Chin. J. Astron. Astrophys. Vol. 0, No. 0, (2006) 000-000

Chinese Journal of

Astronomy and

Astrophysics

\title{
Possible Streams of the Globular Clusters in the Galaxy
}

\author{
Shuang Gao ${ }^{1}$, Bi-Wei Jiang ${ }^{1 \star}$, Yong-Heng Zhao ${ }^{2}$ \\ 1 Department of Astronomy, Beijing Normal University, Beijing 100875 \\ 2 National Astronomical Observatories, Chinese Academy of Sciences, Beijing 100012
}

Received ; accepted

\begin{abstract}
This paper aims to retrieve the ghost streams under the pre-assumption that all the globular clusters in our Galaxy were formed in the very early merge events. The results are based on two speculations: that the specific energy and angular momentum of the globular clusters after merge are not changed in process of evolution and that the globular clusters with common origin would stay in the same orbit plane as parent galaxy. In addition, taking into account the apo-galacticum distance of the orbits, five possible streams were suggested with a significant confidence. The number of streams is consistent with previous results. Three of the four well established members of the Sagittarius stream were found to be in one of our streams. Several other globular clusters in our result were also thought to come from accretion by previous research. Furthermore, the orbital parameters of the streams are derived, which provide a way to testify whether these streams are true with the help of the accurate measurement of proper motions of the globular clusters.
\end{abstract}

Key words: globular clusters: general — Galaxy: formation — Galaxy: halo

\section{INTRODUCTION}

The globular cluster (GC hereafter), as the oldest star group in the universe, has been a target that astrophysics has paid close attention to all the time. The near-field (Galaxy) cosmology has contacted closely with far-field cosmology by the Galaxy's GCs, and the origin of GCs has become an important component of studying the Galaxy's formation and evolution (Freeman \& Bland-Hawthorn 2002).

The earliest view on the Galactic formation is that the halo was created during a rapid collapse of a relatively uniform, isolated protogalactic cloud (Eggen, Lynden-bell \& Sandage 1962). Though this view was challenged (Searle \& Zinn 1978), the theory that GC originate

$\star$ E-mail: bjiang@bnu.edu.cn 
from collapse of the Galactic molecular cloud is stilled insisted by some groups. Harris \& Pudritz (1994) argued that GCs follow a power-law number distribution by mass, $N \sim M^{-1.7}$, which is virtually independent of environment from the study of the connection between protogalactic and present-day cluster formation by examining the GCLF (globular cluster luminosity function) of several large galaxies in addition to the Milky Way.

However, within the scenario of Cold Dark Matter (CDM) cosmology, the universe is built up hierarchically, i.e. the small structure forms early and the large structure forms lately. To our Milky Way galaxy, such scenario fit the view of Searle \& Zinn (1978) that the Galactic halo was built up over an extended period from independent fragments. The discovery of the Sagittarius dwarf galaxy being accreted by the Galaxy supports the view that the halo of the Galaxy has been built up at least partially by the accretion of similar dwarf galaxies (Ibata et al. 1994).

Schweizer (1987) first suspected that GCs were formed in mergers. Later, Ashman \& Zepf (1992) predicted that the Hubble Space Telescope would reveal young GCs which were later discovered in disturbed or interacting galaxies such as NGC 1275 (Holtzman et al. 1992), NGC7252 (Whitmore et al. 1993) and the Antennae (Whitmore \& Schweizer 1995). All these observations support the view that the GCs could be formed in mergers. In our Galaxy, the proof continues. From the Hertzsprung-Russell diagram, Lee et al. (1999) identified multiple stellar populations traced by wide red giant branches in the most massive GC $\omega$ Centauri with different ages and argued that $\omega$ Centauri was once part of a more massive system which merged with the Milky Way. Indeed, Lynden-Bell \& Lynden-Bell (1995) traced the ghostly streams of the outer-halo GCs and satellite galaxies starting from the opinion that they were all formed in early merge events.

Recently, there are several efforts to estimate the number of merges by comparing the characteristics of halo stars or GCs in the Galaxy with those in extra-galaxies. In 1996, Unavane et al (1996) examined the fraction of stars in the halo which have colors consistent with a metalpoor, intermediate-age population matching those typically observed in Local Group dwarf spheroidal galaxies. They concluded that the star counts imply an upper limit of $\sim 60$ merges with low-luminosity dwarf spheroidal, or $\leq 6$ mergers with more luminous objects. In 2000, van den Bergh (2000), based on the survey of young blue halo stars, estimated that the total amount of captured stellar material in the halo is equivalent to 3-7 Sagittarius dwarfs. In 2004, Mackey \& Gilmore (2004) compared in details the properties of GCs between the old halo, young halo and bulge/disk subsystems, and the GCs in LMC, SMC and Fornax and Sagittarius 
dwarf spheroidal galaxies. They estimated the Galaxy may have experienced approximately seven merger events with cluster-bearing galaxies during its lifetime.

In this paper, we try to trace the streams, i.e. the satellite galaxies, of the all the GCs in the halo from their kinetics other than the properties. The analysis presumes that some big satellite galaxies had several GCs which kept their specific energy and angular momentum and which moved in the same orbital plane after merging with our Galaxy. We briefly describe our methods in Section 3 and discuss our results for the possible streams in Section 4.

\section{DATA}

All the parameters of GCs are taken from the web version of "A Catalog of Parameters for GCs in the Milky Way" (Harris 1996). As Harris (2003) had critically selected the best available measurements for each of the quantities included, the quality of the measurements can be regarded at least one of the best. In addition, the electronic version is available through web and updated regularly, which guarantees the data being the latest.

The catalog is consisted of three parts, including positions, photometric parameters and kinetic parameters respectively. It lists the cluster identifications, positions, integrated magnitudes, colors, morphology parameters, metallicities, radial velocities, and structural parameters. We make use of the parameters such as radial velocities, positions and distances. However, the necessary parameters are not fully available for 3 GCs among the 150 GCs in the Harris catalog because they are faint, inside the Galactic plane, or lately found. So our analysis is directed to the 147 GCs for which the needed parameters are complete without consideration of the errors of the parameters.

\section{METHOD AND CALCULATION}

Two major criteria are set to sort the common origin of GCs. The first criterion is that the GCs of common origin should have the same specific energy and angular momentum. The second one is that their orbits should lie within the same plane. At the beginning, all the GCs are treated equally nevertheless they are different in some characteristics such as metallicities or morphologies of horizonal branches which are usually used in classification. Specifically, they were all assumed to come from the accreted galaxies even if some of them were originally

produced in the Galaxy. However, the final result from our analysis will screen out the members which might be Galactic natives. 


\subsection{Hypotheses}

To proceed our analysis, there are several strong hypotheses as following which are essentially the same as those by Lynden-Bell \& Lynden-Bell (1995).

- Sufficiently large satellite galaxies have GCs in their own halos. Such GCs would be pulled off if the satellite galaxies were tidally torn. Indeed, it's this hypothesis that motivated us to search for the Galactic GCs of common parent galaxies, though the origin of the GCs in the large satellite galaxies is another question.

- In tidal tearing, the objects torn off pursue orbits in the same plane through the Galactic center as their parent's orbit.

- The objects were torn off at one or several close passages during a period in which the energy and angular momentum of the progenitor's orbit did not change very much.

- The initial orbits of the objects torn off had approximately the same specific energy and specific angular momentum as the progenitor.

- The gravitational potential in the Galactic halo has changed only slowly since that time and may now be taken to have a known form.

\subsection{Specific Energy and Angular Momentum}

As mentioned in the beginning of this section, the first criterion of the GCs from the same parent galaxy is their equal specific energy and angular momentum of the GCs. The specific energy $E$ and the angular momentum $h$ are defined to be the total energy and angular momentum per unit mass respectively. The equation of specific energy is as follows:

$$
E_{r}=\frac{1}{2} v_{r}^{2}-\psi=E-\frac{1}{2} h^{2} r^{-2}
$$

where $E_{r}$ is the radial component of total energy, $r, \psi$ and $v_{r}$ are the Galactocentric distance, gravitational potential and Galactocentric radial velocity respectively.

According to equation (1), the GCs with the same specific energy and angular momentum can be found out, if knowing the gravitational potential of the halo, $\psi$, Galactocentric distance, $r$, and the velocity $v_{r}$, because the GCs of the same origin, which have the same specific energy and angular momentum, are expected to lie on the same line delineated by equation (1) with the gradient $-h^{2} / 2$ and intercept $E$. Thus, it is necessary to determine $\psi, r$ and $v_{r}$ to proceed.

1. First, the gravitational potential $\psi$ is calculated. Through the rotation curve of the Galaxy, we have the law that the density in the Galactic halo vary with Galactocentric 
distance, thus have the equation of the gravitational potential. Assuming the density is positive everywhere and falls like $r^{-2}$ for $r \ll r_{h}$, but like $r^{-5}$ for $r \gg r_{h}$, the form of the potential can be given by

$$
\psi=V_{0}^{2} \ln \left(\frac{\sqrt{1+S^{2}}+1}{S}\right),
$$

where $V_{0}^{2}=220 \mathrm{~km} \mathrm{~s}^{-1}$ is the circular velocity of the Sun, $S=r / r_{h}$ and $r_{h}=80 \mathrm{kpc}$. Thus, for a given $r$ which is the Galactocentric distance, the potential can be calculated.

2. The Galactocentric distance $r$ can be calculated from the solar distance and the Galactic coordinates from simple trigonal relation.

3. From the distant halo, the difference in direction of the Sun and Galactic center can often be neglected, so the radial velocity in the line of sight, $v_{l}$ (after correction to the Galactic center of rest), is approximately the radial velocity that would be seen from the Galactic Center, $v_{r}$. Thus $v_{r}$ can be regarded as known. It should be kept in mind that this approximation is virtually valid for the GCs distant from the Galactic center and will bring about some uncertainty for those relatively close to the Galactic center.

From the available data, 147 of all the 150 GCs have been measured in $v_{r}^{2}$ and $r$. The diagram of their radial energy $E_{r}$ vs Galactocentric distance $r$ is shown in Fig. 1, where the solid curve describes the variation of the potential $\psi$ with $r$.

To determine the straight lines on Fig. 1 through which some of the diffuse 147 points pass, a method called "Hough Transform (hereafter HT)" is adopted. Because there are 147 points on Fig. 1, the number of possible lines is $C_{147}^{2}$ by connecting any two of the points. The HT method essentially calculates all the slopes and intercepts of these $C_{147}^{2}$ lines and finds out those lines which have close slopes and intercepts, therefore the points making these lines should have similar specific energy and angular momentum.

The analysis showed that the gathering of the slopes of lines were relatively concentrated while that of the intercepts were scattering, thus grouping the specific energy and angular momentum considered the error in the range of intercepts. As an example shown in Fig. 2, the $1 \sigma$ errors are plotted with the upper line and lower line being the error bounds. Consequently, the points within $1 \sigma$ error were regarded to have the same specific energy and angular momentum, i.e. passing the first criterion examination. This analysis of the 147 points yielded 21 lines, which means there are 21 groups with the same $E$ and $h^{2}$. The names and number of the members in each group are shown in Table1. The streams in Table 1 are sorted by the intercepts of the lines decreasing from top to bottom, which is indeed the specific total energy $E$. 
It can be seen from Table 1 that the number of members of each group ranges from 3 to 14. Indeed, more than 3 points on a line is required to make a group. As an example, one of the 21 lines is displayed in Fig. 2, where the points in the same group are between the upper line and lower line.

\subsection{Polar-path Maps}

The objects satisfying the second criterion, i.e. moving in the same orbits, is selected through the Lambert equal-area projection. Different from the traditional projection, in Lambert projection, the x-y plane's transform equations are following:

$$
\begin{aligned}
& x^{\prime}=\frac{x}{\sqrt{1+z}}, \\
& y^{\prime}=\frac{y}{\sqrt{1+z}},
\end{aligned}
$$

where $\left(x^{\prime}, y^{\prime}\right)$ are coordinates transformed from the $\mathrm{x}-\mathrm{y}$ plane. After such transformation, the size of projected area is proportional to and thus retains the original relative sizes, which is very important in present work because only its position is known for a given GC.

The poles of the countless orbits resulted from the limited information (i.e. the position of the GC and that the Galactic center is within the orbital plane) consist a set which is called polar-path. The equation of the polar-path is described in the Cartesian coordinates by:

$$
x^{2}\left(1+\frac{\cos ^{2} l}{\tan ^{2} b}\right)+y^{2}\left(1+\frac{\sin ^{2} l}{\tan ^{2} b}\right)+2 x y \frac{\sin l \cos l}{\tan ^{2} b}=1,
$$

where $(l, b)$ are the Galactic coordinates of the given GC.

The search for the common orbits is converted to search for the intersection points of the polar paths of the GCs. For every of the 21 groups in Table 1, the polar-path is plotted for each GC in the projected plane. Whether the polar-paths have intersections, which was judged by eyes, demonstrates whether they have the same orbit. It is found that all or part of the members have intersections in eight groups, i.e. they have the same orbits. while no more than 3 members have intersections in thirteen groups which are regarded not to move in the same orbit.

These eight groups for which some members are found to have polar-path intersections are listed in Table 2, as well as the NGC serial numbers of the members with common poles of orbital planes (some members in Table 1 are dropped due to lack of intersection with others). As examples, the polar paths of the members in two groups are shown in Figure 3, where the left panel indicates a successful one, the eighth group in the 21 streams in Table 1 , and the right panel displays the failed search, number 16 in Table 1. 


\subsection{Error Analysis}

During the process to search for the groups, some errors are induced from approximations or methods. Firstly, the Galactocentric radial velocity is approximated by the line-of-sight velocity. This approximation is true only for the objects which are very distant from the Galactic center where the position difference between the Sun and the Galactic center has little effect on the velocity difference. It brings about significant error in the case of nearby GCs. Secondly, the error is induced from applying the HT method to pick out the points on one line. These errors

have been utilized to restrict the ranges of selecting points, and have been plotted on Fig.2 (dashed lines). Because there are uncertainties in the parameters of velocity and distance of GCs, we suffered some dispersion in the transformed slope-intercept diagram (see Section 3.2 for details) of the points. This means that the members in the same group may have some scattering in the specific energy and angular momentum. Thirdly, the common poles from the Lambert's equal-area projection are not exactly at one point, but with some range. Whether the GCs have the same orbital pole is judged by eyes, with the difference in $X^{\prime}$ and $Y^{\prime}$ less than 0.1 or so.

\section{RESULT AND DISCUSSION}

\subsection{The Orbital Parameters}

From the analysis of the specific energy and angular momentum, and the polar path of the GCs, we found 8 groups, with the members in each group possibly having common satellite origin. Furthermore, based on the position parameters of the members and the pole position as the intersections in Fig. 3 in each of the eight streams, the equation of the normal to each orbital plane was solved. The orbital parameters of the 8 groups are shown in Table 3, where the stream number (first appearing in Table 1), the apo-galacticum, peri-galacticum, eccentricity, specific angular momentum, specific total energy, period and coefficients of the orbital equation are listed from left to right. The ambiguity of the direction of the normal to the orbital plane brings about two combinations of the coefficients. That's why the last two columns have two combinations. This degeneracy can be relieved by estimating the direction of the angular momentum, which will be carried out in future more detailed work.

It can be seen from Table 3 that the streams no. 18, 19, and 21 have their apo-galacticum less than $8.5 \mathrm{kpc}$, the adopted solar distance to the Galactic center. For the members in these streams, the approximation of the solar radial velocity to the Galactic radial velocity could bring about large error in calculating the specific energy and angular momentum. Moreover, 
the GCs with the orbit so close to the Galactic center should have been disrupted due to the strong tidal force if they had been accreted long before. Another disfavor of these three objects is that one object (NGC 5904 and NGC 6558) appears in two streams, which indicates mediocre quality in grouping. Therefore, these three streams should not be the ghost streams, which are separated by a line in Table 3 from the candidate streams. Finally, the five streams, specifically no. 5, 6, 8, 9 and 10, are possible streams of the GCs in the Galaxy from the kinetic point of view.

The eccentricity of the GCs's orbits has been discussed widely because it is related to their origins as well as the evolution. Statler(1988) suggested that the orbital eccentricity of GCs is decreasing in the process of dissipative through numerical simulation method. Gnedin(2006) derived the average eccentricity of surviving model clusters to concentrate between 0.4 and 0.8. This is consistent with the early result by Ninković (1983). In Table.3, the eccentricities of possible streams are between 0.5 to 0.8 , which agrees well with the high probability eccentricities of GCs. The result indirectly confirms our calculation.

\subsection{Number of Streams}

From our analysis, there are five streams for the origin of about 20\% GCs in the Galaxy, which means there had been five relatively large and cluster-bearing satellite galaxies being accreted in early times. This number is well consistent with others. Unavane et al. (1996) concluded $\leq 6$ mergers with luminous galaxies. van den Bergh (2000) estimated the total amount of captured stellar material equivalent to 3-7 Sagittarius dwarfs. Mackey \& Gilmore (2004) estimated the Galaxy may have experienced approximately seven merger events with cluster-bearing galaxies during its lifetime. All these results agree reasonably well with ours.

\subsection{The Sagittarius Stream}

As the best established stream, the Sagittarius stream has been investigated in most details. Consequently, some GCs have been suggested as the members of the Sagittarius stream. The discovers Ibata et al. (1997) suggested NGC 6715 (also known as M54), Ter 8, Ter 7 and Arp 2 being associated with the Sagittarius stream. Bellazzini et al (2003) added Pal 12, NGC 4147. The 2MASS color-magnitude diagram (Bellazzini et al. 2004) enhanced their belief that the two GCs are the members. Pal. 12 (Martínez-Delgado et al. 2002) was also proposed ro be a member. Although numerous additional members have been postulated, there are only four well-established members as suggested by Ibata et al. (1997). From Table 2, stream no.6 includes three of the four established members, i.e. NGC 6715, Ter 8 and Ter 7, which is in 
good coincidence with previous results. The left member Arp 2 does not appear in this group, which may be caused by the error in our analysis or that it is not the member from the kinetic point of view. The other postulated members neither appear in stream no.6. In addition, the other member in stream no.6 is IC-1276 which is in the Galactic bulge (Barbuy et al. 1998), and thus possibly is not the member. We conclude that stream no.6 is at least part of the Sagittarius stream.

\subsection{Other Members}

In addition to the members of the Sagittarius stream, several more GCs in our streams also appear in previous identifications. NGC 7492, NGC 7089, NGC 6809 and NGC 5904 which appear in stream no. 5, 10, 18 and 19 (though no. 18 and 19 may not be the stream due to their close orbit to the Galactic center) were suggested to be similar to GCs in extragalaxies by Mackey \& Gilmore (2004). Pal 5 which appears in stream no. 8 was considered to lie in a stream from the SDSS-II data (Odenkirchen et al. 2003). Our result confirmed some GCs speculated to originate externally. However, the exact streams are difficult to configure. Besides, some of the GCs in our results have not been found to be of external origin by other methods. Because the method in our analysis suffers some uncertainty (especially lack of the accurate measurement of proper motions) and that the judgement only from the properties of GCs is not decisive, further investigations are needed to clarify the origins of the GCs in the Galaxy.

\section{SUMMARY}

The present work agree with that the Galaxy had experienced several merges. Our result classified the GCs of possible external origin into 5 groups, which is well consistent with previous results. In particular, three of the four well established Sagittarius members were classified into one stream. As previous work identified the external origin of GCs mainly from their observed properties such colors, metallicity or age, our work identified their origin from their kinematic properties. Since our view is different from others, the consistent conclusion is important.

Whether the stream found is true or imaginary needs further investigations, even if the uncertainties mentioned above could all be eliminated. This is because the features - the same specific angular momentum and the same orbit plane - can not guarantee the GCs originated from external galaxies, i.e., the GCs of the same origin inside the Galaxy would also preserve such characteristics. However, other properties will help to judge if our sort of groups is right or wrong. For example, the accurate determination of the GCs' proper motions will make 
it possible to calculate the three dimensional velocities and thus the right orbits. Presently available data and observational techniques can yet provide such data. The launch of GAIA (Mignard 2005) will provide high precision measurements of proper motions to Galactic objects.

We expect then the test of our results.

ACKNOWLEDGMENTS We thank the anonymous referee who provided a helpful critique of the manuscript. This research was supported by NSFC under grant 10573022, and the Undergraduate Research Foundation of Beijing Normal University.

\section{REFERENCES}

Ashman K.M., Zepf S.E, 1992, ApJ, 384, 50

Barbuy B., Ortolani S., Bica E., 1998, A\&AS, 132, 333B

Bellazzini M., Ibata R., Ferraro F., 2004, In: F. Prada, D. Martinez, T.J. Mahoney, eds, ASP Conf. Ser. Vol. 327, Satellites and Tidal Streams, San Francisco: ASP, p. 220

Bellazzini M., Ibata R., Ferraro F., Testa V., 2003, A\&A, 405, 577

Eggen O.J., Lynden-Bell D., Sandage A.R., 1962, ApJ, 136, 748 (ELS)

Freeman K., Bland-Hawthorn J., 2002, ARA\&A, 40, 487

Gnedun Y., Prieto L., 2006, In: T. Richtler, et al eds, Invited Review for Conference "Globular Clusters, Guide to Galaxies", Chile: University of Concepcion

Harris E, Pudritz E., 1994, ApJ, 429, 177

Harris E., 1996, AJ, 112, 1487

Harris, W. 2003, available at http://www.physics.mcmaster.ca/resources/globular.html and http://www.mporzio.astro.it/ marco/gc/

Holtzman, J., Faber, S., Shaya, E. et al., 1992, AJ, 103, 691

Ibata R., Gilmore G., Irwin M., 1994, Nature, 370, 194

Ibata R., Wyse R., Gilmore, G. et al., 1997, AJ, 113,, 634

Lee Y.W., Joo J.M., Sohn Y.J. et al., 1999, Nature, 402, 55

Lynden-Bell D., Lynden-Bell R.M., 1995, MNRAS, 275, 425

Mackey A., Gilmore G., 2004, MNRAS, 355, 504

Martínez-Delgado D., Zinn R., Carrera R., et al., 2002, ApJ, 573, L19

Mignard F., 2005, In: Turon C., O'Flaherty K., Perryman M., eds, the Observatoire de ParisMeudon, The proceedings of the Gaia Symposium "The Three-Dimensional Universe with Gaia", SP 576, 5, Paris: ESA

Ninković S., 1983, Anstron. Nachr., 304, 305

Odenkirchen M., Grebel Eva K., Dehnen W., et al. 2003, AJ, 126, 2385 O

Schweizer, F. 1987, in Nearly Normal Galaxies: From the Planck Time to the Present, ed. Faber, S., 18

Searle L., Zinn R., 1978, ApJ, 225, 357

Statler S., 1988, ApJ, 331, 71

Unavane M., Wyse R., Gilmore G., 1996, MNRAS, 278, 727

van den Bergh S. 2000, ApJ, 530, 777

Whitmore B., Schweizer F., Leitherer C., et al., 1993, AJ, 106, 1354

Whitmore B., Schweizer F. 1995, AJ, 109, 960 
Table 1 The intermediate results from the specific angular momentum and energy of all the 147 GCs, including 21 streams. The columns are: stream serial number, number of members in the stream, the members in the stream with the NGC serial number, or the name if not included in the NGC catalogue.

stream $\sharp$ Counts Members' ID(NGC)

\begin{tabular}{|c|c|c|}
\hline 1 & 5 & 65396453635258247492 \\
\hline 2 & 3 & 671258247492 \\
\hline 3 & 7 & 6517 Lynga 76496622958247492 Terzan 3 \\
\hline 4 & 9 & 63626656674959863201622958247492 Terzan 3 \\
\hline 5 & 10 & 6544 Pal 1065844590622958247492 Terzan 369346981 \\
\hline 6 & 7 & IC 127641476715 Terzan 8 Terzan 769346981 \\
\hline 7 & 6 & Pal 867526101185154665634 \\
\hline 8 & 8 & 6517 Lynga 7625412611904 Pal 5 IC 1257 Pal 12 \\
\hline 9 & 5 & 64026535649662056864 \\
\hline 10 & 14 & 6426686452722808708968386584 Pal 10636267606656621865406388 \\
\hline 11 & 4 & 288 Terzan 1264536539 \\
\hline 12 & 8 & 288707810468386496653563166712 \\
\hline 13 & 9 & 6779104683852866584 Pal 1061446341362 \\
\hline 14 & 8 & 52864372 Pal 1062546496634161716553 \\
\hline 15 & 6 & 64416352437270995897 Pal 11 \\
\hline 16 & 7 & Liller $16235614464966356 \mathrm{Pal} 115897$ \\
\hline 17 & 6 & 635659464833628451396809 \\
\hline 18 & 8 & 64406681680959464833628451395904 \\
\hline 19 & 8 & Terzan 16558594648336284513959046362 \\
\hline 20 & 7 & 6254665664966760654467496333 \\
\hline 21 & 6 & 671264536558635565536171 \\
\hline
\end{tabular}


Table 2 The final results include 8 groups in which part or all members have a common orbital poles. The columns are the same as Table 1. The GCs which were regarded coming from accretion by other research are labeled and the corresponding references are shown at the footnotes. See the text for details.

\begin{tabular}{|c|c|c|c|c|c|c|}
\hline Stream $\sharp$ & Counts & Member & ' ID $(\mathrm{NC}$ & iC) & & \\
\hline 5 & 3 & Pal-10 & $7492^{\ddagger}$ & 6934 & & \\
\hline 6 & 4 & IC-1276 & $6715^{*}$ & Ter-7* & Ter- $8^{*}$ & \\
\hline 8 & 5 & 6517 & 6254 & Pal $-5^{\dagger}$ & Pal-12 & IC- 1257 \\
\hline 9 & 3 & 6402 & 6535 & 6864 & & \\
\hline 10 & 4 & 5272 & $7089^{\ddagger}$ & 6838 & Pal-10 & \\
\hline 18 & 4 & 6440 & 6681 & $6809^{\ddagger}$ & $5904^{\ddagger}$ & \\
\hline 19 & 4 & Ter-1 & 6558 & $5904^{\ddagger}$ & 6284 & \\
\hline 21 & 5 & 6453 & 6558 & 6355 & 6553 & 6171 \\
\hline
\end{tabular}

*Ibata et al. 1997

$\dagger$ Odenkirchen M. et al. 2003

$\ddagger$ Mackey \& Gilmore 2004

Table 3 The results with the 8 orbital parameters and the quality of result. The $r_{\max }$ and $r_{\min }$ are the orbital apo-galacticum and peri-galacticum distances respectively, $e$ is the orbital eccentricity, $P$ is orbital period, $a$, $b$ and $c$ are the parameters of the normal to orbital plane delineated by $a x+b y+c z=0$.

\begin{tabular}{ccccccccc}
\hline Stream\# & $\frac{r_{\max }}{k p c}$ & $\frac{r_{\min }}{k p c}$ & $e$ & $\frac{10^{-3} h}{k p c \cdot k m / s}$ & $\frac{-10^{-5} E}{(k m / s)^{2}}$ & $\frac{P}{10^{8} y r}$ & $\frac{b}{a}$ & $\frac{c}{a}$ \\
\hline 5 & 29.88 & 5.50 & 0.69 & 2.2414 & 0.8 & 3.11 & $\mp 0.78$ & 0 \\
6 & 24.84 & 2.72 & 0.80 & 1.2604 & 0.9 & 2.76 & $\mp 0.39$ & $\mp 2.31$ \\
8 & 19.15 & 4.00 & 0.65 & 1.5868 & 1.0 & 1.96 & $\mp 3.08$ & $\mp 0.84$ \\
9 & 15.21 & 3.73 & 0.64 & 1.4142 & 1.1 & 1.45 & $\mp 2.34$ & \pm 0.29 \\
10 & 14.75 & 4.46 & 0.54 & 1.5868 & 1.1 & 1.51 & $\mp 0.71$ & $\mp 0.02$ \\
\hline 18 & 6.84 & 1.21 & 0.70 & 0.5018 & 1.5 & 0.71 & $\mp 9.69$ & \pm 0.64 \\
19 & 6.73 & 1.42 & 0.65 & 0.5630 & 1.5 & 0.69 & \pm 58.29 & \pm 1.03 \\
21 & 3.49 & 0.97 & 0.57 & 0.3552 & 1.8 & 0.35 & $\mp 64.38$ & \pm 3.94 \\
\hline
\end{tabular}




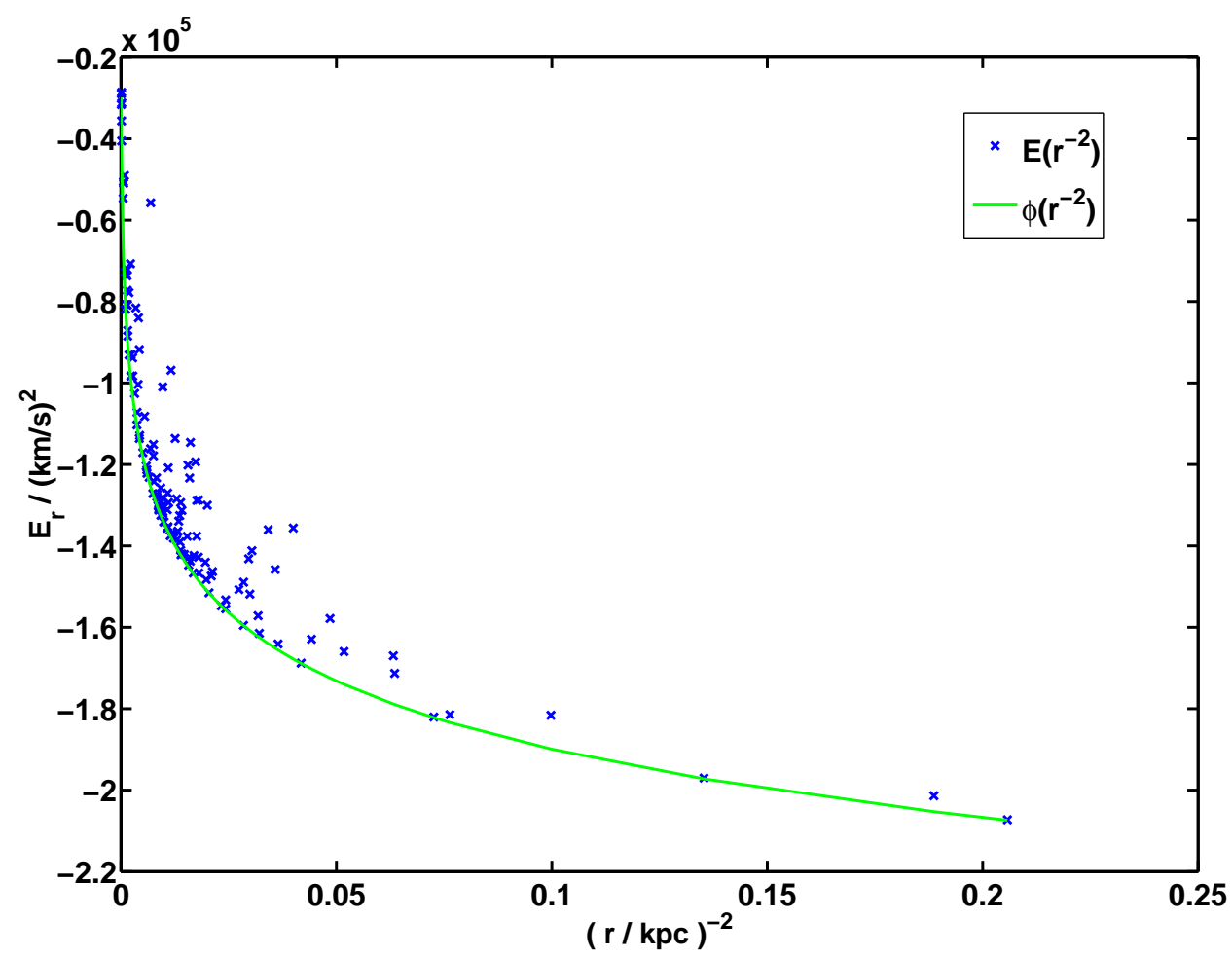

Figure $1 \quad E_{r}=\frac{1}{2} v_{r}^{2}-\psi$ plotted against $r^{-2}$. Members of one stream should lie on one straight line in the diagram.

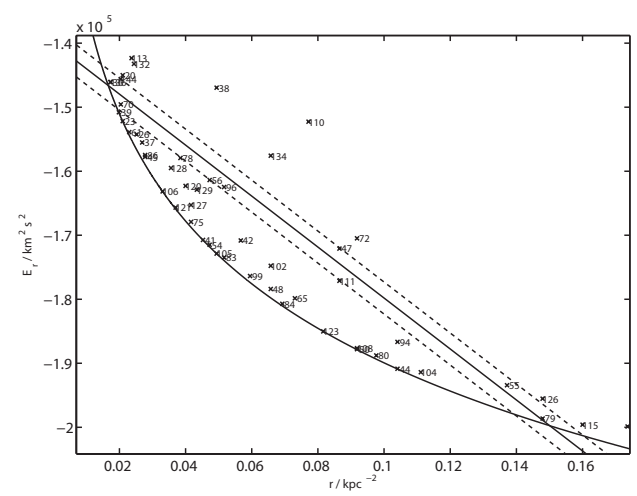

Figure 2 One line in the radial energy diagram. There are 7 points between the upper line and lower line. These 7 objects is classified as Stream no. 16 in Table 1. The cross denotes a GC and the digit beside labels the sequence number of the corresponding GC in the Harris table. 

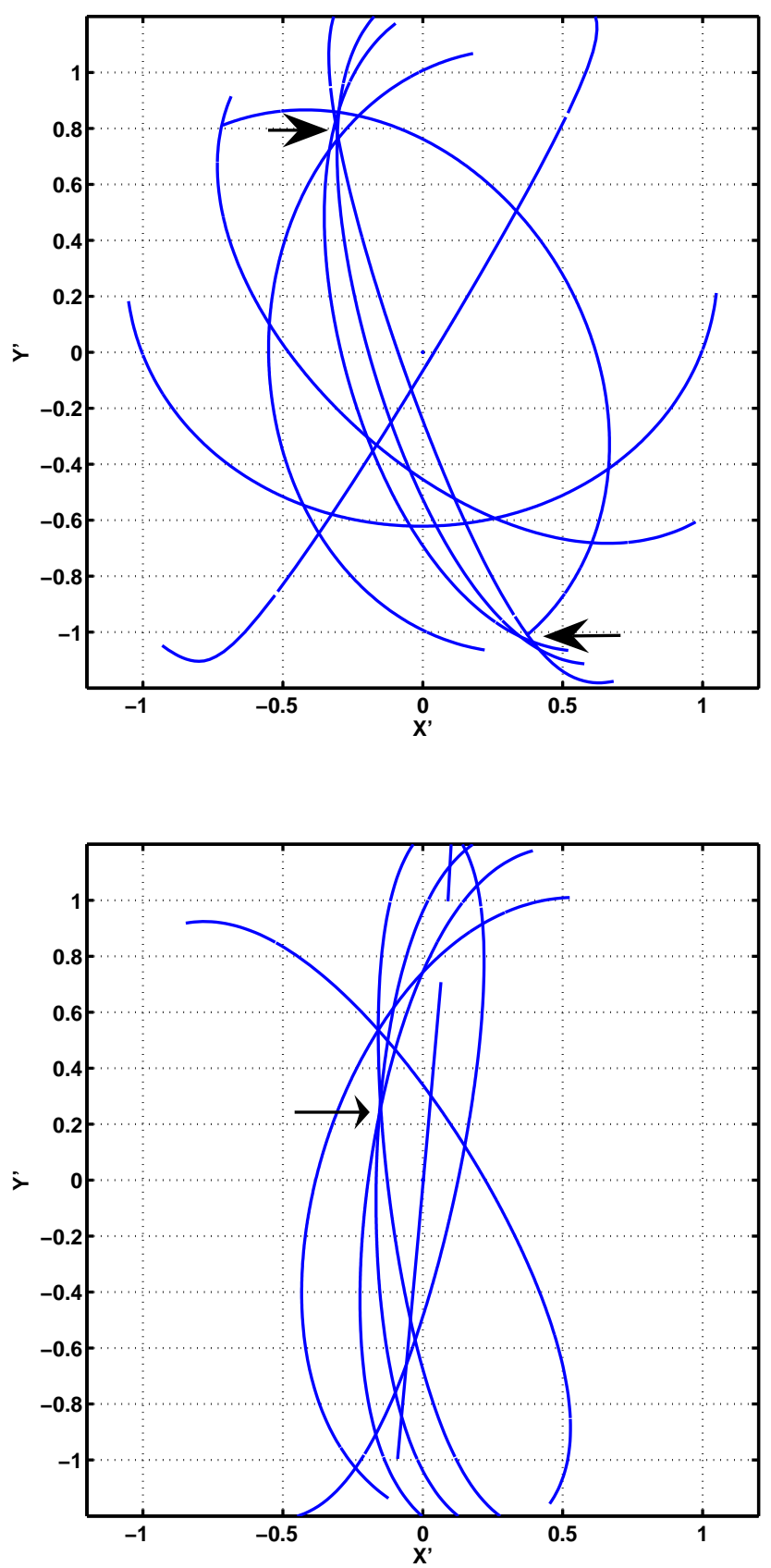

Figure 3 Polar paths of two groups of globular clusters. The left: the members of stream no. 8 streams in Table1. The right: the members of stream no. 16 in Table1. Each path is a great circle perpendicular to the galactocentric vector to the globular cluster, $X^{\prime 2}+Y^{\prime 2}=1$ (see text for details) is the Galactic plane. $X^{\prime 2}+Y^{\prime 2}<1$ is the northern Galactic sky. Multiple intersections of polar paths means possible stream poles. The arrow in the diagram points out these poles. 